\title{
THE THEORY OF NUMBERS
}

An Introduction to the Theory of Numbers By Prof. G. MI. Hardy and Prof. E. M. Wright. Pp. xvi +404 . (Oxford: Clarendon Press; London : Oxford University Press, 1938.) 25s, net.

THE theory of numbers has an appeal to general interest, not shared by other branches of mathematics, in that most of the great unsolved problems of the subject are formulated in terms intelligible to everybody. There must be many people of limited mathematical attainments who have felt attracted to the subject, but whose interest has died away for the want of just such a book as this to stimulate it.

The point of view from which the book is written can best be described in the authors' own words.

"It is not in any sense (as an expert can see by reading the table of contents) a systematic treatise on the theory of numbers. It does not even contain a fully reasoned account of any one side of that many sided theory, but is an introduction, or a series of introductions, to almost all of these sides in turn. We say something about each of a number of subjects which are not usually combined in a single volume, and about some which are not always regarded as forming part of the theory of numbers at all. . . We have often allowed our personal interests to decide our programme, and have selected subjects less because of their importance (though most of them are important enough) than because we found them congenial and because other writers have left us something to say."

There can be no doubt that the authors have carried out their programme with marked success. In spite of their modest disclaimer, the book is really as systematic as any elementary account of so extensive a subject can be, or ought to be. It presupposes, for the most part, no more knowledge of mathematics than is possessed by a first. year university student, and yet it introduces the reader to almost all branches of the theory of numbers, including some which have only been developed in recent times. Naturally, in some branches, the deeper results have to be stated without proof; but the book does contain proofs of practically all theorems that are genuinely elementary (using the word in its non-technical sense). Mathematicians of all kinds will find the book pleasant and stimulating reading, and even experts on the theory of numbers will find that the authors have something new to say on many of the topics they have selected.

The selection of topics, and the general approach to the subject, hare a slight analytical bias, as will be seen from the list of contents. The reader is introduced at the outset to the $O, O$ and $\sim$ notation of modern analysis, and later to the notions of 'almost all integers', 'sets of measure zero', and 'uniform distribution'.

Each chapter is a model of clear exposition, and the notes at the ends of the chapters, with their references and suggestions for further reading, are invaluable. The only points in the book which the reviewer feels any inclination to criticize are (1) the section on integral quaternions, where the exposition of Dickson has been preferred to the more elegant theory of Hurwitz; (2) the definition of a convex area, which is obscured by the mention of a convex curve (a convex area or body is a much simpler thing than a curve, let alone a simple, closed, continuous curve); (3) the absence of an explicit theorem on the existence of primitive roots $(\bmod p)$.

The printing is excellent, and has not that squashed appearance which is such a common fault of present-day English mathematical printing.

In the following summary of the contents, chapters which deal with the same side of the subject are grouped together.

Chapters i, ii, xxii (first half): the series of primes. A fairly complete account of what is known and what is conjectured about the distribution of the primes, with proofs where these are of an elementary character.

Chapters v, vi, vii, viii : congruences. The classical elements of the theory of numbers.

Chapters iv, ix, $x, x i$, xxiii : Diophantine approximation. Accounts are given of decimals and continued fractions, proofs of the transcendence of $e$ and $\pi$, and three proofs of Kronecker's theorem.

Chapters xii, xiii, xiv, xv : quadratic fields. A few particular fields are treated, and the field $k(\sqrt{-3})$ is used to prove the insolubility of the equation $x^{3}+y^{3}=z^{3} \quad(x y z \neq 0)$.

Chapters xvi, xvii, xviii, xxii (second half): arithmetical functions. The formal properties of various arithmetical functions are considered, together with the associated Dirichlet series, also the order of magnitude, average order of magnitude, and normal order of magnitude of some of them.

Chapters xix, $\mathrm{xx}, \mathrm{xxi}$ : additive theory. One chapter is devoted to partitions, one to representations by two or four squares, and one to Waring's problem. The chapter on partitions is one of the most attractive in the book.

Chapters iii, xxiv: geometry of numbers. Minkowski's theorems on lattices and linear forms.
H. Davenrort. 